\title{
Enzyme-Substrate Interactions Revealed by the Crystal Structures of the Archaeal Sulfolobus PTP-Fold Phosphatase and its Phosphopeptide Complexes
}

\author{
Hsing-Mao Chu ${ }^{1,2}$ and Andrew H.-J. Wang ${ }^{1,2 *}$ \\ ${ }^{1}$ Institute of Biochemical Sciences, National Taiwan University, Taipei 106, Taiwan \\ ${ }^{2}$ Institute of Biological Chemistry, Academia Sinica, Taipei 115, Taiwan
}

\begin{abstract}
The P-loop-containing protein phosphatases are important regulators in signal transduction. These enzymes have structural and functional similarity with a conserved sequence of $D x(25-41)$ HCxxGxxR(T/S) essential for catalysis. The singular protein tyrosine phosphatase (PTP) from archaeal Sulfolobus solfataricus is one of the smallest known PTPs with extreme thermostability. Here, we report the crystal structure of this phosphatase and its complexes with two tyrosyl phosphopeptides A-(p)Y-R and N-K(p)Y-G-N. The structure suggests the minimal structural motif of the PTP family, having two variable sequences inserted between the $\beta 2-\beta 3$ and $\beta 3-\beta 4$ strands, respectively. The phosphate of both phosphopeptide substrates is bound to the P-loop through several hydrogen bonds. Comparison of several phosphatase-substrate complexes revealed that Gln135 on the Q-loop has different modes of recognition toward phosphopeptides. The substrate specificity of SsoPTP is primarily localized at the phosphotyrosine, suggesting that this phosphatase may be a prototypical PTP. Proteins 2007;66:996-1003. ๑ 2006 Wiley-Liss, Inc.
\end{abstract}

Key words: phosphatase; synchrotron crystallography; mutagenesis; P-loop; sulfolobus; hyperthermophilic; phosphotyrosine

\section{INTRODUCTION}

The regulation of cellular processes via protein phosphorylation-dephosphorylation is a ubiquitous and prominent mechanism. This is coordinated by the opposing actions of protein tyrosine kinase (PTK) and protein tyrosine phosphatase (PTP). Both enzymes have been evolutionarily conserved among eukaryotes, bacteria, and archaea. ${ }^{1,2}$ In archaea, the phosphorylation of protein tyrosine residues was recently confirmed by the finding of phosphoproteins in Sulfolobus solfataricus, Haloferax volcanir, and Methanosarcina thermophila. ${ }^{2}$ The first archaeal phosphoprotein to be identified was the methyltransferase activation protein from the methanogenic archaeon Methanosarcina barkeri. More recently, three tyrosine-phosphorylated proteins have been isolated and identified from lysates of the hyperthermophilic archaeon Thermococcus kodakaraensis. ${ }^{3}$ These three proteins are involved in RNA, glycogen, and amino acid metabolism, respectively, and the reactions are controlled by PTPs.

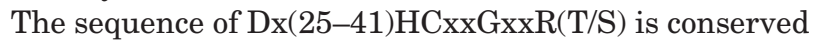
in PTPs and dual specificity phosphatases (DSPs), where $\mathrm{x}$ is any residue and the conserved cysteine residue is required for catalysis, acting as a nucleophile in the catalytic mechanism. ${ }^{4}$ In addition to the conserved active-site motif, both the PTPs and DSPs also have a conserved aspartic acid residue that functions as a general acid. Genome searches revealed the presence of over 50 ORFs whose predicted products contained the $\mathrm{HCx}_{5} \mathrm{R}$ sequence in prokaryotes with known genome sequences, and thus are likely to encode functional PTP/DSPs. Interestingly, several archaeal genomes have widespread existence of several "eukaryotic" and "bacterial" protein kinases and phosphatases ${ }^{5}$ and the number of protein kinases in archaea usually exceed those of protein phosphatases. Especially in $M$. thermoautotrophicum, P. horikoshii, and S. solfataricus, the ratio of potential Ser/Thr/Tyr protein kinases to potential countervailing Ser/Thr/Tyr protein phosphatases is quite high $(4: 1) .^{5}$ In $S$. solfataricus, there are seven potential protein kinases, whereas only two protein phosphatases (one PP1-arch and one PTP) are found. ${ }^{2}$ This implies that protein phosphorylation in this archaeal organism may be unevenly regulated by kinases and phosphatases.

To date only one archaeal PTP has been characterized, TkoPTP from T. kodakaraensis, ${ }^{3}$ but no crystal structure

Abbreviations: DSP, dual-specificity protein phosphatase; PMM, phosphomannosemutase; pNPP, para-nitrophenylphosphate; PTP, protein tyrosine phosphatase; PTP1B, protein tyrosine phosphatase 1B; r.m.s.d., root mean square deviation.

The Supplementary Material referred to in this article can be found at http://www.interscience.wiley.com/jpages/0887-3585/suppmat

Data deposition: The structure factors and atomic coordinates reported in this paper have been deposited in the Protein Data Base, www.rcsb.org (PDB ID codes 2I6I, 2I6J, 2I6M, 2I6P, 2DXP and 2I60).

Grant sponsor: Academia Sinica; Grant sponsor: Core Facility for X-ray Crystallographic Research, Taiwan; Grant number: NSC943112-B-001-010-Y.

*Correspondence to: Andrew H.-J. Wang, Institute of Biological Chemistry, Academia Sinica, Taipei 115, Taiwan.

E-mail: ahjwang@gate.sinica.edu.tw

Received 11 July 2006; Revised 30 August 2006; Accepted 15 September 2006

Published online 15 December 2006 in Wiley InterScience (www. interscience.wiley.com). DOI: $10.1002 /$ prot.21262 
of archaeal PTP has been solved. Genomic searching showed that there is only one potential PTP in the genome of $S$. solfataricus. Herein, we report the first structural study of this primitive $S s o$ phosphatase (SsoPTP) which provides functional insights of archaeal phosphatases in hyperthermophilic species. Our study showed that recombinant SsoPTP exhibited phosphohydrolase activity toward $p N P P$, phosphotyrosine, and phosphopeptides (A-(p)Y-R and N-K-(p)Y-G-N) in vitro. The results also helped us trace the structural origin and the evolution of the conserved PTP domain. To the best of our knowledge, this is the first report on the structural features of an archaeal PTP.

\section{MATERIALS AND METHODS Sample Preparation and Crystallization}

S. solfataricus phosphatase (161 amino acids) gene was cloned into the pET21b (Novagen) vector and overexpressed in Escherichia coli BL21 (DE3, RIL) (Novagen). The protein was purified from Ni-NTA column with $25 \mathrm{~m} M$ Tris (pH 7.5), $150 \mathrm{mM} \mathrm{NaCl}$, and $300 \mathrm{~m} M$ imidazole and concentrated to $14 \mathrm{mg} / \mathrm{mL}$ by Amicon centrifugal concentrator (Millipore), using the membrane with $5000 \mathrm{Da}$ molecular weight cut-off. Mutant protein (C96S) was prepared by using the QuikChange ${ }^{\mathrm{TM}}$ SiteDirected Mutagenesis Kit (Stratagene) according to manufacturer's instructions.

For crystallization experiments, the hanging-drop vapor diffusion method was used, with the protein-toreservoir ratio being $1 \mu \mathrm{L}: 1 \mu \mathrm{L}$ for wild-type and mutant proteins. With Wizard I crystallization kits for the initial screening, wild-type crystals was obtained with $0.1 \mathrm{M}$ citrate ( $\mathrm{pH} 5.5$ ) and 20\% PEG 3000. Crystallization conditions for SsoPTP-phosphate complex were the same as those used for the wild type apo enzyme, except that the buffer was $0.2 M$ sodium/potassium phosphate $(\mathrm{pH} 5.5)$ instead of $0.1 M$ citrate. For the tungstate complex, $5 \mathrm{mM}$ sodium tungstate was added to the crystallization buffer. Cocrystals of phosphatase with $p N P P$ were obtained with the reservoir containing $0.1 M$ Hepes $(\mathrm{pH} 7.5)$ and $35 \%$ PEG 600. Crystallization conditions for the C96S mutant were $0.1 M$ MES ( $\mathrm{pH}$ 6.5) and $12 \%$ PEG 20000, soaked with phosphopeptides A-(p)Y-R or N-K-(p)Y-G-N (final $3.5 \mathrm{mM}$ ). All crystals were grown at room temperature and the reservoir volume was $500 \mu \mathrm{L}$. Crystals were soaked for $5 \mathrm{~min}$ in a cryoprotectant containing $25 \%$ glycerol before data collection.

\section{Data Collection and Structure Determination}

Diffraction data were recorded using a Rigaku R-Axis $\mathrm{IV}^{++}$detector mounted on an in-house Rigaku rotatinganode X-ray generator. The data sets of crystal form $\mathrm{P} 4_{1}$ of the native crystal was collected at Taiwan beamline BL12B2 in SPring-8, Japan, using the ADSC Quantum 4R charge-coupled device. The data were processed and integrated with DENZO and SCALEPACK. ${ }^{6}$ The detailed statistics are listed in Table I. The crystal structure of the wild-type SsoPTP was determined by the molecular replacement method $^{7}$ using model of Cdc14B (PDB 1OHE) with the data of 50-1.9 $\AA$ range. The final refined model contains full length protein (residues 1-161) with clear electron density. Model building and refinement were performed with XtalView ${ }^{8}$ and CNS program. ${ }^{9}$ Improvement of the model was guided by the Sigma A-weighted $2 F_{\mathrm{o}}-F_{\mathrm{c}}$ electron density maps. Other structures were similarly determined and refined. The molecular figures were produced using MOLSCRIPT, ${ }^{10}$ RASTER3D, ${ }^{11}$ and GRASP. $^{12}$

\section{Phosphatase Assays}

The standard reaction that contained $1 \mu \mathrm{g}$ of the phosphatase, $50 \mathrm{~m} M$ Tris- $\mathrm{HCl}(\mathrm{pH} 6.0)$, and $10 \mathrm{~m} M p$-nitrophenyl phosphate $(p N P P)$ in a final volume of $1 \mathrm{~mL}$ was performed at $37^{\circ} \mathrm{C}$ for $20 \mathrm{~min}$ and then stopped by addition of $\mathrm{NaOH}$ to the final concentration of $0.1 M$. The increase in the amount of $p$-nitrophenol released by the enzymatic degradation of $p$ NPP was monitored by measuring the absorbance at $420 \mathrm{~nm}$ on an Ultraspec 4000 (Pharmacia) spectrophotometer. The pH-dependent phosphatase activity was determined by incubating the standard reaction mixture at various $\mathrm{pH}$ conditions at $37^{\circ} \mathrm{C}$.

\section{RESULTS AND DISCUSSION Overall Structure of the SsoPTP}

The overall structure of SsoPTP reveals a compact PTP fold composed of a slightly twisted five-stranded $\beta$ sheet, surrounded by eight helices $(\alpha \mathrm{A}-\alpha \mathrm{H})$ [Fig. 1(A)]. A search using DALI ${ }^{13}$ showed that its homologous structures include $\mathrm{Cdc} 14 \mathrm{~B},{ }^{14} \mathrm{KAPt},{ }^{15}$ and $\mathrm{VHR}^{16}$ members of the PTP family. Structural similarities are mainly found in the region having secondary structural elements. Although the topology of SsoPTP is similar to that of the catalytic domain of Cdc14B (sharing $32 \%$ sequence identity and root-mean-square deviation of $1.17 \AA$ using 512 backbone atoms by Swiss-PdbViewer program $)^{17}$ [Fig. S2(B)], substantial differences are also found in several regions (two loop regions between the $\beta 2-\beta 3$ strands and the $\beta 3-\beta 4$ strands to be discussed later).

We have determined the structures of the wild type phosphatase and its complexes with phosphate, tungstate, as well as the inactive C96S variant in complex with $p N P P$ and two phosphopeptides, (A-(p)Y-R and N-K-(p)Y-G-N) [Fig. 1(A,B) and S1.(A)], from which we could evaluate the substrate recognition. In the $S s o \mathrm{PTP}-p N P P$ complex, the phosphate of $p N P P$ is bound deeply in the active site with eight hydrogen bonds. The phenyl ring of $p N P P$ interacts with the hydrophobic side chains of Val97 and Ile100, and with the backbone of the P-loop. The interactions induce a conformational change of the P-loop that tightens the binding of $p N P P$ with the P-loop. Meanwhile, binding of $p N P P$ leads to Gln135 interacting with the oxygen of $p N P P$, 
TABLE I. Data Collection and Refinement Statistics

\begin{tabular}{|c|c|c|c|c|c|c|}
\hline Data collection & SsoPTP & $\begin{array}{l}\text { SsoPTP + } \\
\text { phosphate }\end{array}$ & $\begin{array}{l}\text { SsoPTP + } \\
\text { tungstate }\end{array}$ & $\begin{array}{c}\mathrm{C} 96 \mathrm{~S}+ \\
p N P P\end{array}$ & $\begin{array}{l}\text { C96S + } \\
\text { A(p)YR }\end{array}$ & $\begin{array}{c}\text { C96S + } \\
\text { NG(p)YKN }\end{array}$ \\
\hline Source & $\mathrm{Cu} \mathrm{K} \alpha$ & SPring-8 BL12B2 & $\mathrm{Cu} \mathrm{K} \alpha$ & $\mathrm{Cu} \mathrm{K} \alpha$ & $\mathrm{Cu} \mathrm{K} \alpha$ & $\mathrm{Cu} \mathrm{K} \alpha$ \\
\hline \multicolumn{7}{|l|}{ Unit cell } \\
\hline$a(\AA)$ & 72.36 & 72.29 & 72.29 & 34.20 & 81.54 & 80.98 \\
\hline$b(\AA)$ & 72.36 & 72.29 & 72.29 & 57.65 & 58.23 & 58.40 \\
\hline$c(\AA)$ & 32.92 & 32.44 & 32.52 & $\begin{array}{c}42.52 \\
\beta=92.87\end{array}$ & 36.48 & 36.44 \\
\hline Space group & $\mathrm{P} 4_{1}$ & $\mathrm{P} 4_{1}$ & $\mathrm{P} 4_{1}$ & $\mathrm{P} 2{ }_{1}$ & $\mathrm{P} 2{ }_{1} 2_{1} 2$ & $\mathrm{P} 2_{1} 2_{1} 2$ \\
\hline \multirow[t]{2}{*}{ Resolution $(\AA)^{\mathrm{a}}$} & 2.15 & 1.66 & 1.9 & 2.5 & 2.1 & 1.9 \\
\hline & $(2.15-2.23)$ & $(1.72-1.66)$ & $(1.97-1.9)$ & $(2.59-2.5)$ & $(2.18-2.1)$ & $(1.97-1.9)$ \\
\hline Observed $^{\mathrm{b}} /$ & $22,140^{\mathrm{b}} /$ & $8,0837^{\mathrm{b}} /$ & $29,556^{\mathrm{b} /}$ & $15,524 /$ & $4,2621^{\mathrm{b}} /$ & $69,445^{\mathrm{b}} /$ \\
\hline $\begin{array}{l}\text { unique } \\
\text { reflections }\end{array}$ & 8,759 & 19,655 & 12,144 & $5,618(F>2 \sigma)$ & 10,072 & 13,698 \\
\hline Completeness (\%) & $91.9(77.9)$ & $97.5(81.8)$ & $89.7(73.2)$ & $96.9(91.7)$ & $94.2(91.3)$ & $96.2(92.6)$ \\
\hline Average $I / \sigma(\mathrm{I})$ & $18.8(3.9)$ & $25.1(2.3)$ & $12.6(1.7)$ & $17.6(2.8)$ & $17.0(2.8)$ & $17.5(2.5)$ \\
\hline$R_{\text {merge }}(\%)$ & $6.1(19.9)$ & $6.6(46.0)$ & $9.0(55.5)$ & $7.7(31.9)$ & $7.7(44.7)$ & $9.0(60.7)$ \\
\hline \multicolumn{7}{|l|}{ Refinement } \\
\hline $\begin{array}{l}R / R_{\text {free }}(\%)^{\mathrm{c}} \\
\text { r.m.s.d }\end{array}$ & $21.9 / 25.0$ & 20.9/24.1 & $22.3 / 23.6$ & $22.4 / 28.6$ & $21.1 / 25.2$ & $19.1 / 22.6$ \\
\hline Bond lengths $(\AA)$ & 0.008 & 0.005 & 0.006 & 0.007 & 0.006 & 0.005 \\
\hline $\begin{array}{l}\text { Bond angles } \\
\text { (degree) }\end{array}$ & 1.503 & 1.448 & 1.349 & 1.338 & 1.304 & 1.238 \\
\hline \multicolumn{7}{|l|}{$\begin{array}{l}\text { Average } \\
\quad \text { B-factor }\left(\AA^{2}\right)\end{array}$} \\
\hline $\begin{array}{l}\text { Protein/ } \\
\quad \text { substrate } / \mathrm{H}_{2} \mathrm{O}\end{array}$ & $19.5 /-/ 32.1$ & $17.8 / 19.8 / 34.2$ & $22.1 / 63.8 / 24.2$ & $48.5 / 54.8 / 31.9$ & 26.4/43.5/39.6 & $18.0 / 29.2 / 34.9$ \\
\hline
\end{tabular}

${ }^{a}$ Values in parentheses indicate the specific values in the highest resolution shell.

${ }^{\mathrm{b}}$ Reflection, $F>0 \sigma$.

${ }^{\mathrm{c}} R_{\text {free }}=\mathrm{R}$ factor calculated using $5.0 \%$ of the reflection data chosen randomly and omitted from the start of refinement.

instead of interacting with the carbonyl of Gly132 as found in the apo phosphatase [Fig. S1(B)].

We chose two phosphopeptides as the possible substrates. The substrate A-(p)Y-R sequence contains a basic arginine, potentially capable of binding to the acidic surface of the phosphatase, whereas the N-K-(p)Y-G-N sequence is a predicted phosphorylation site of a possible phosphoprotein substrate, phosphomannosemutase (PMM). ${ }^{3}$ In the SsoPTP-N-K-(p)Y-G-N complex structure, the phosphopeptide backbone approximates an extended $\beta$-strand [Fig. 1(C)]. The phosphate of pTyr is tightly bound by the P-loop and the phenyl ring of the pTyr residue is partly buried within the active site, surrounded by the Ploop, Asp69 of the D-loop, and Gln135 of the Q-loop [Fig. 1(D)]. The carboxylate group of the general acid Asp69 donates a hydrogen bond to the $\mathrm{O}$ atom of the pTyr. These interactions between pTyr and the residues of the active site are similar to those of other phosphatases. In addition, we observed water-mediated interactions between Arg130 and the $\operatorname{Arg}(\mathrm{P}+1)$ residue of $\mathrm{A}-(\mathrm{p}) \mathrm{Y}-\mathrm{R}$ [Fig. 1(B,E)], or the P-loop and the Gly $(\mathrm{P}+1)$ residue of N-K-(p)Y-G-N [Fig. $1(C, D)]$, suggesting that the phosphatase may interact with substrate proteins using varied water-mediated networks. Other notable hydrogen bonds, contributed by the Gln135 residue of the Q-loop, included the contribution of bidentate hydrogen bonds from the side chain of Gln135 to the carbonyl and amide groups of the Asn (P-2) residue of
N-K-(p)Y-G-N and the interaction with the Ala(P-1) residue of A-(p)Y-R [Fig. 1(C)]. These hydrogen bonds are important in orienting the peptides. The detailed interactions of the SsoPTP complexed with two phosphopeptides are shown in Figure 1(E,F).

However, the binding modes found in the SsoPTPphosphopeptide complexes differ from those observed for phosphotyrosine peptide recognition by $\mathrm{PTP}^{18} \mathrm{~B}^{18}$ and phosphothreonine peptide recognition by KAPt. ${ }^{15}$ The side chain of the conserved Gln181 was rotated away from the catalytic site relative to the KAPt-sulfate complex to avoid the steric hindrance with the pThr residue. Interestingly, we found opposite actions of the Gln135 residue between the SsoPTP-phosphate and SsoPTP$p N P P$ complexes. The side chain of Gln135 in the Q-loop is pointed out of the catalytic site in the SsoPTP-phosphate complex, but faces the catalytic site in the SsoPTP-pNPP complex.

\section{Differences in Active Site Interactions}

When we compared the SsoPTP-A(p)Y-R with SsoPTP-N-K-(p)Y-G-N complexes, we found that they have some deviations toward the $\mathrm{N}$-terminal residues [Fig. 2(A)]. We further compared our SsoPTP-phosphopeptide complexes with other complexes of classical 

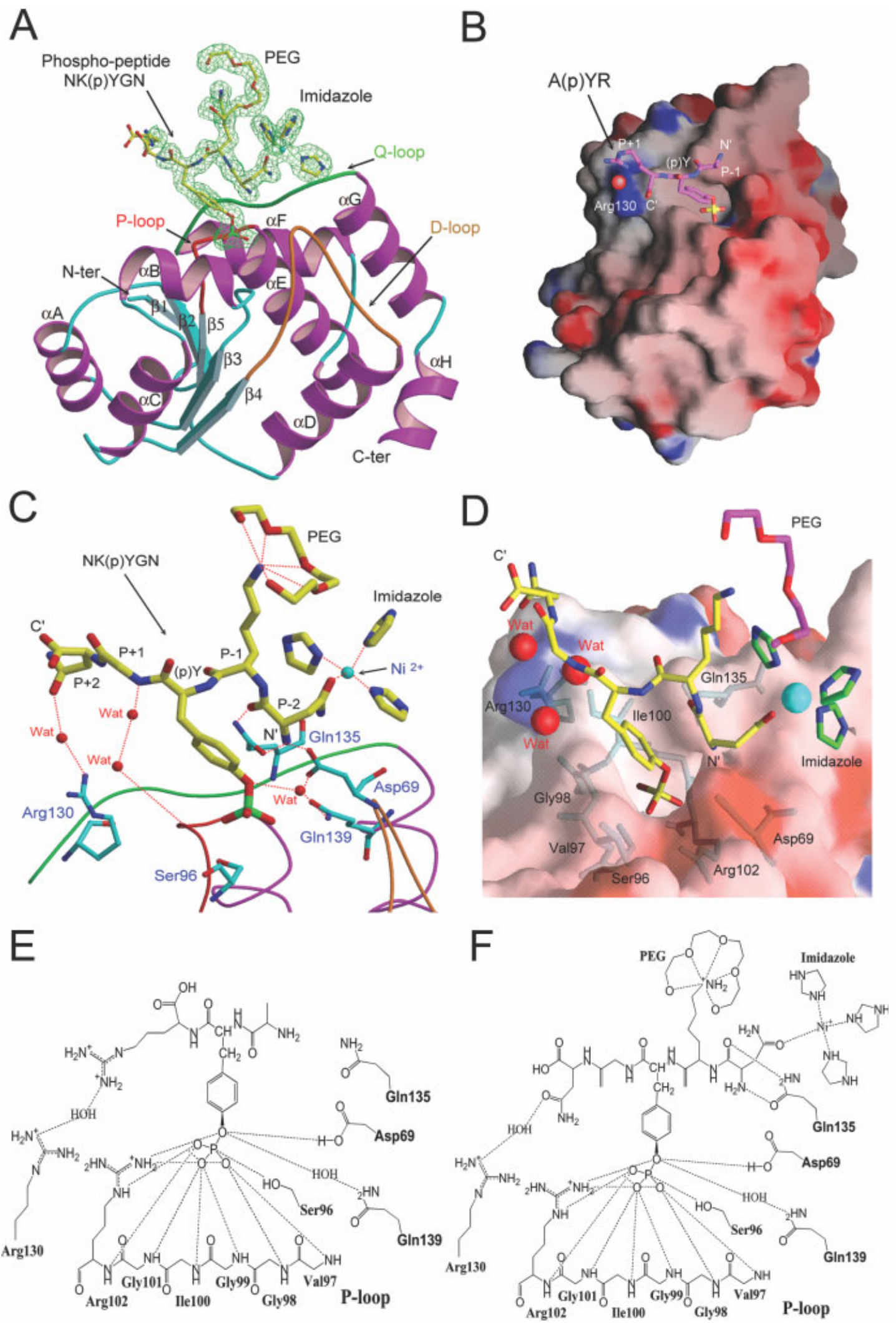

Fig. 1. Structures of the SsoPTP in complex with phosphopeptides. (A) The view showing the overall structure of the SsoPTP-phosphopeptide (N-K-(p)Y-G-N) complex. The P-loop, D-loop, and Q-loop are in red, yellow, and green, respectively. The phosphopeptide substrate is shown as yellow color, and the density is $2 F_{\mathrm{o}}-F_{\mathrm{c}}$ map contoured at $1.0 \sigma$. (B) A GRASP electrostatic surface representation of the complex of the SsoPTP with phosphopeptide (A-(p)Y-R) potentials ranging from $-15 \mathrm{eV}$ (red) to $+15 \mathrm{eV}$ (blue). (C) Details of the interactions between phosphopeptide (N-K(p)Y-G-N), PEG, metal ion and the SsoPTP. (D) An enlarged figure of surface potential shows the active site of the SsoPTP-N-K-(p)Y-G-N complex. $(\mathrm{E})$ and $(\mathrm{F})$ Schematic diagrams of interactions involved in A-(p)Y-R and N-K-(p)Y-G-N binding with SsoPTP. 

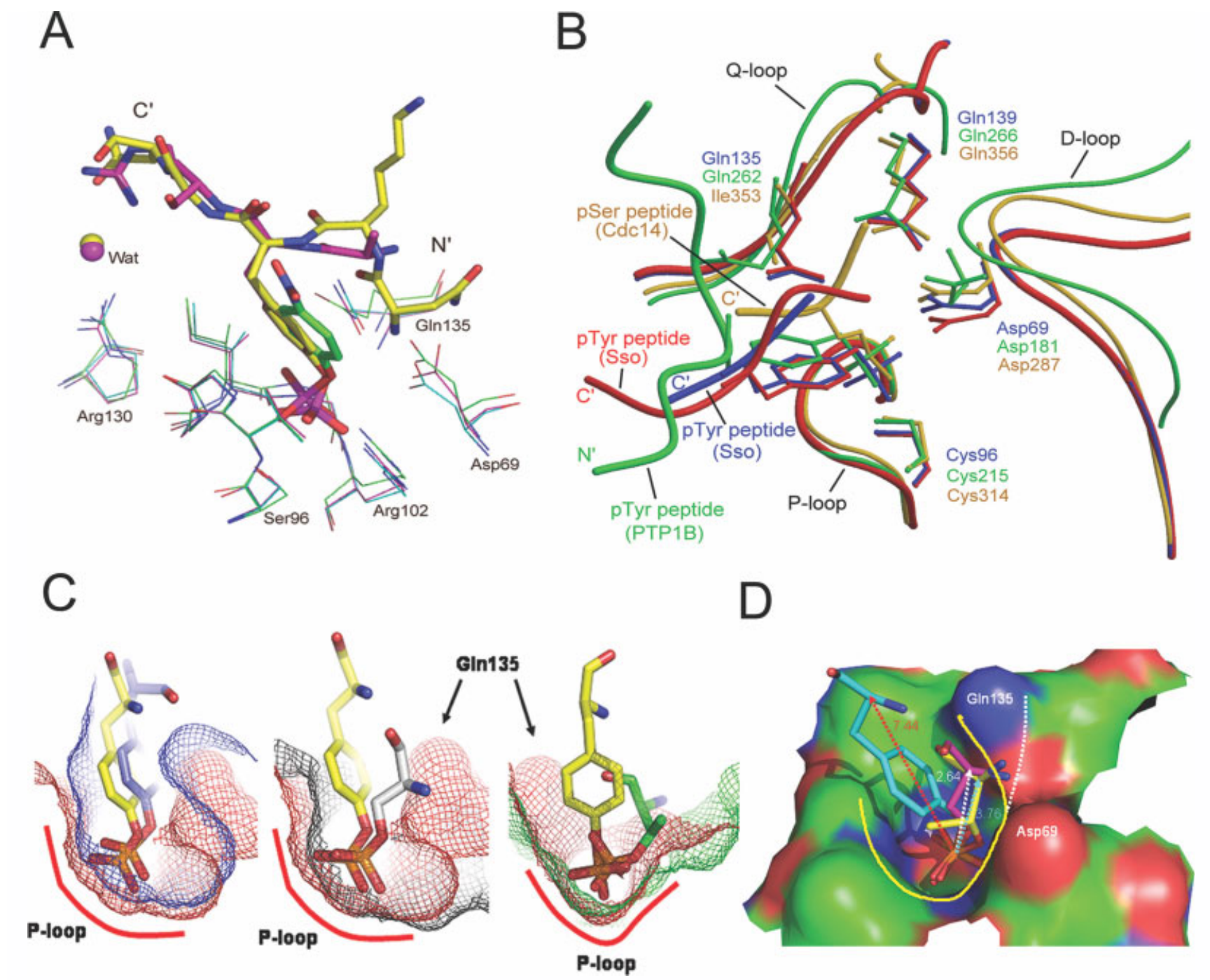

D

Fig. 2. Structural comparison of different complexes of phosphatases. (A) Comparison of the complex structures of the SsoPTP with $p N P P$ (magenta), A-(p)Y-R (green) and N-K-(p)Y-G-N (cyan). (B) Comparison of SsoPTP-phosphopeptide complex (cyan), Cdc14B-phosphopeptide complex (yellow) and PTP1B-phosphopeptide complex (magenta). (C) The superimposed comparison of the binding pocket of SsoPTP (red) with PTP1B (blue), Cdc14B (dark) and KAPt (green), respectively. (D), We put the three phosphor-residues in the active site, and found that the shape of the pocket of the SsoPTP is most adaptive to fit substrate pTyr (The length of red arrow: 7.44 A), rather than the shorter pSer (The length of white arrow: $2.64 \AA$ ), or pThr (The length of cyan arrow: $3.76 \AA$ ) residues. The yellow line represents the direction of opening of the pocket as same as the direction of red arrow. The white line represents the wider pocket to accommodate the pSer or pThr residues.

PTP $^{19}$ (e.g., PTP1B) and DSPs (e.g., Cdc14 and KAPt). We found that the backbone orientation of the bound phosphopeptide (N- to C-terminus) [Fig. 2(B)] in the SsoPTP complexes is different from that of the PTP1B-phosphopeptide complex, but similar to those in two DSP (Cdc14 and KAPt) complexes. In addition, the depth of the active site pocket is always considered to be a key determinant of phospho-amino acid substrate specificity. ${ }^{20}$ So far, the phosphatase specificity toward either pTyr, pSer, or pThr by sequence alignment alone can not be predicted. In general, the active site of the classical PTP is deeper than that of DSP in order to accommodate the bulky pTyr residue at the active site.

The pocket shape of the SsoPTP active site indicated a better fit for the pTyr substrate (7.44 $\AA$ ), as opposed to the shorter pSer $(2.64 \AA)$ or pThr (3.76 ̊) residues [Fig. 2(D)]. The Gln135 residue is a critical structural feature in hindering the entry of either the pSer or the pThr residues. Because of the sloped angle of the backbone of the pSer or pThr residues, the binding pocket for these residues is shallow and, thus, the direction of the substrate peptide backbone [drawn in white line, Fig. 2(D)] is opposite to that for the pTyr peptide [drawn in yellow line in Fig. 2(D)]. This structural observation complements the results of our enzymatic assay in that SsoPTP is only active for the pTyr substrate. A comparison of the binding pocket of SsoPTP with PTP1B, Cdc14B, and KAPt shows that KAPt displays the broadest substrate binding pocket while that of SsoPTP is between that of PTP1B and Cdc14B [Fig. 2(C)]. 
A

\begin{tabular}{|c|c|c|c|c|c|c|c|}
\hline & $\begin{array}{c}\text { Sso } \\
\text { phosphatase }\end{array}$ & VHY & PTP1B & Cdc14B & $\mathrm{KAPt}$ & PTEN & Phytase \\
\hline \multirow[t]{2}{*}{ Box1 } & & & & & & & \\
\hline & $16 \mathrm{AA}$ & 16AA & $19 \mathbf{A A}$ & 30 $\mathbf{A A}$ & $27 \mathbf{~ A A}$ & $30 \mathbf{A A}$ & $23 \mathrm{AA}$ \\
\hline \multirow[t]{2}{*}{ Box 2} & & & & & & & \\
\hline & $25 \mathbf{A A}$ & $11 \mathbf{A A}$ & 59 AA & $16 \mathrm{AA}$ & $24 \mathrm{AA}$ & $14 \mathbf{A A}$ & 81AA \\
\hline
\end{tabular}

\section{B}
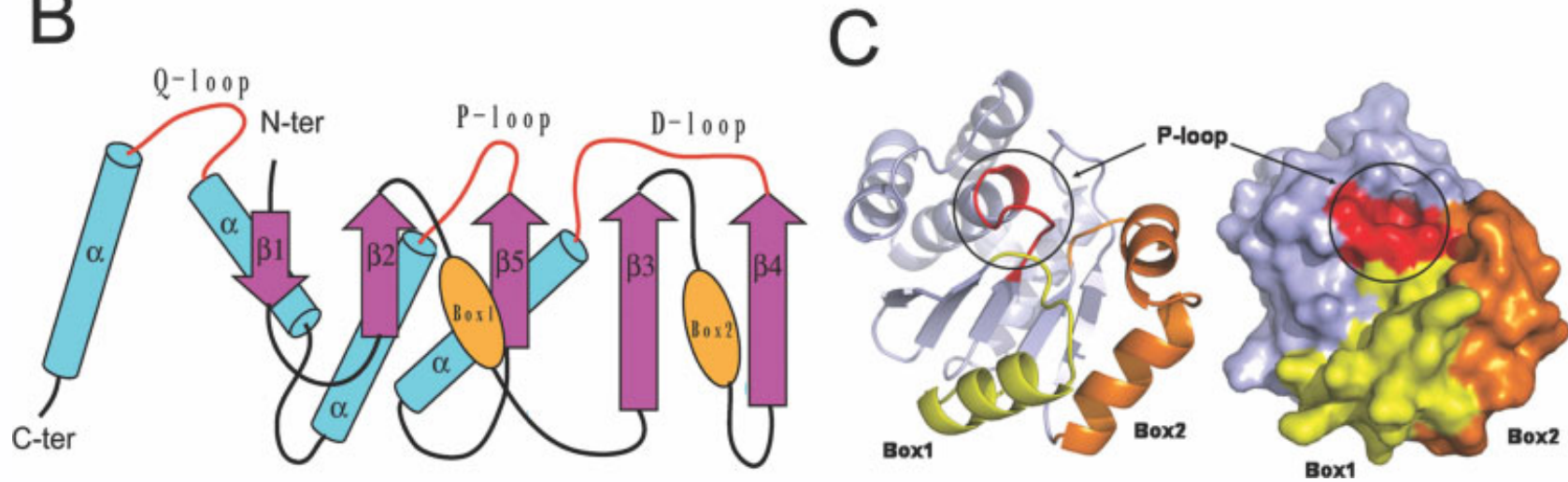

Fig. 3. Comparison of the topology diagram of DSP fold phosphatases. (A) The topology of the SsoPTP. (B) The structural features of P-loop containing phosphatases divided into a most conserved region and two diverse loop regions (Box1 and Box2). (C) Ribbon and surface diagrams of SsoPTP reveal the region of Box1 and Box2, which is associated with the major diversity in many PTPS and DSPs.

\section{Two Variable Sequence Regions in PTPs}

Through sequence and structure alignment of several $\mathrm{P}$ loop-containing phosphatases, including SsoPTP, VHY, ${ }^{21}$ PTP1B, Cdc14B, KAPt, PTEN, ${ }^{22}$ and phytase, ${ }^{23}$ a core structure containing a five stranded $\beta$ sheet and four $\alpha$-helices could be identified. However, within these phosphatases, sequences between strands $\beta 2-\beta 3$ and $\beta 3-\beta 4$ are highly variable in length and structure [Fig. 3(A)]. We may define the two "variable inserts" located between strand $\beta 2-\beta 3$ and strand $\beta 3-\beta 4$ as Box1 and Box2, respectively [Fig. 3(B)]. The structure of Box1 is an $\alpha$-helix (helix $\alpha 1$ ) in SsoPTP, and we found that the region influences the conformation of the P-loop through the interaction of the main-chain amide group of Leu14 on helix $\alpha 1$ with the main-chain carbonyl group of Val97 of the P-loop. Box2 is a helix-loop-helix structure in SsoPTP. This region serves the important function of orienting the phosphate-binding arginine side-chain in the P-loop either through the mainchain carbonyl (in the case of VHR) or through side-chain hydrogen bonds (Glu115 of PTP1B). Hence, Glu40 of
SsoPTP may play a similar role through interacting with the Arg102 of P-loop.

When we compared the two regions of Box1 and Box2 within several PTP members, we found that Box1 is basically an $\alpha$-helix structure in SsoPTP, PTEN, and PTP1B, and the length of helix ranges from 16 aa (SsoPTP) to 30 aa (PTEN). The length of $\alpha$-helix of Cdc14B in Box1 is similar to that in SsoPTP, but contains an additional loop. In $\mathrm{KAPt}$, a $\beta / \alpha$ mixed structure exists in Box1.

Box2 is more divergent than Box1. In SsoPTP and KAPt, Box2 is a helix-loop-helix. However, in PTP1B, there is a complicated all- $\beta$ structure. In PTEN, Box2 is a 14 aa random coil structure. From a structural point of view, it is possible to build the smallest PTP-fold phosphatase without any insertion in Box1 and Box2. By searching the NCBI protein database, we found many small P-loop containing phosphatases [Fig. S2(A)] and, possibly, the smallest PTP-like fold phosphatase of 141 amino acids from $B$. cereus. A model of $B$. cereus phosphatase was built through homology modeling using 
SsoPTP as a template. Structural comparison of $S s o$ and $B$. cereus phosphatases show that $B$. cereus phosphatase adopts the simplest structural element in both Box1 (13 aa) and Box2 (13 aa) [Fig. S2(C)].

In conclusion, Box 1 and Box 2 are found to surround the surface of active site cleft, and the arrangement of these two divergent regions of the phosphatase could create different interacting surfaces in order to bind to different substrate proteins [Fig. 3(C)]. This observation may explain the substrate specificity of PTP-like fold phosphatases.

\section{Interactions of SsoPTP with Ligands}

Interestingly, in our SsoPTP-N-K-(p)Y-G-N complex structure, we found that PEG molecule, $\mathrm{Ni}^{2+}$ ion, and imidazole molecules are unexpectedly co-crystallized with the complex [Fig. 1(A)]. The side chain of Lys residue in (N-K-(p)Y-G-N) is stabilized by the PEG molecule in ways similar to those between cyclic crown ether and ammonium ion. In addition, the interactions among $\mathrm{Ni}^{2+}$ ion, imidazole molecules, and the side chain of the Gln residue of the phosphopeptide are analogous to the metal ion-mediated interactions in proteins, which help anchor the charged side chain of the substrate phosphopeptides on the enzyme.

\section{Analysis of the Phosphatase Activity}

The phosphotyrosine dephosphorylation activities of the wild-type enzyme were examined using para-nitrophenol phosphate (an analog of free phosphotyrosine) as the substrate. SsoPTP displays its optimal phosphatase activity at $\sim 90^{\circ} \mathrm{C}$ and $\mathrm{pH}$ of $\sim 4.5$ [Fig. S3(A,B)]. It also exhibited phosphohydrolase activity toward phosphotyrosine and two phosphopeptides (A-(p)Y-R and N-K-(p)Y$\mathrm{G}-\mathrm{N}$ ) in vitro. Finally, in order to test for other phosphoresidues, we measured the phosphatase activities on phosphoserine and phosphothreonine. However, SsoPTP did not release inorganic phosphate from either phosphoserine or from phosphothreonine. Thus, functionally, SsoPTP apparently belongs to the PTP family.

\section{Possible Function of SsoPTP}

SsoPTP exhibits structural and functional features similar, but not identical, to those of eukaryotic enzymes. For structural features, comparative analysis of several kinds of phosphatase-phosphopeptide complexes suggests that although SsoPTP belongs to the PTP family according to its enzymatic activity, the binding mode of SsoPTP with its substrate is more similar to that of DSPs. In addition, because of the lack of sequence-specific interactions between the enzyme and peptide substrates, SsoPTP can bind with different peptides through water-mediated interactions and to the Gln135 residue of the Q-loop. Hence, according to our structural analysis in the present study and from previous biochemical data, we propose a model for the interaction between SSoPTP and PMM (Fig. S4). The substrate specificity is primarily localized at the phosphorylated Y298, with the Gln135 and Asp69 residues of SsoPTP making several interactions with PMM. Box1 and Box2 lack direct contact with PMM.

In prokaryotes, the high ratio of potential PTKs to potential countervailing PTPs is interesting. ${ }^{5}$ SsoPTP may recognize many phosphoproteins to control different biological processes. The SsoPTP-phosphopeptide complexes reveal the structural basis of the plasticity of the active site in substrate recognition and are also similar to those of PTP1B-phosphopeptides complexes. ${ }^{24}$ These characteristics may be beneficial to the binding of many phosphoproteins in vivo, and hence evolutionally, the usage of minimal number of the phosphatases may provide the growth advantage in the extreme environments of the hyperthermophilic archaeal species.

In conclusion, the crystal structure of SsoPTP reveals that it is a PTP with the bare minimal structure of the PTP/DSP fold. There are many phosphotyrosine-containing proteins in S. solfataricus, ${ }^{3,25}$ but analysis of the genomic database of $S$. solfataricus suggests that only one tyrosine phosphatase is encoded in the genome. Taken together, these points permit us to suggest that the lone phosphatase in S. solfataricus might be a general PTP capable of acting on many different phosphoproteins.

\section{ACKNOWLEDGMENTS}

The synchrotron data collections were conducted with the Biological Crystallography Facilities (Taiwan Beamline BL12B2 at SPring-8, Japan) supported by the National Science Council (NSC).

\section{REFERENCES}

1. Zhang ZY. Protein tyrosine phosphatases: structure and function, substrate specificity, and inhibitor development. Annu Rev Pharmacol Toxicol 2002;42:209-234.

2. Shi L, Potts M, Kennelly PJ. The serine, threonine, and/or tyrosine-specific protein kinase and protein phosphatases of prokaryotic organisms. A family portrait. FEMS Microbiol Rev 1998; 22:229-253.

3. Jeon SJ, Fujiwara S, Takagi M, Tanaka T, Imanaka T. TK-PTP, protein tyrosine/serine phosphatase from hyperthermophilic archaeon Thermococcus kodakaraensis KOD1: enzymatic characteristics and identification of its substrate proteins. Biochem Biophys Res Commun 2002;295:508-514.

4. Denu JM, Dixon JE. A catalytic mechanism for the dual-specific phosphatases. Proc Natl Acad Sci USA 1995;92:5910-5914.

5. Kennelly PJ. Archaeal protein kinase and protein phosphatase: insights from genomics and biochemistry. Biochem J 2003;370: 373-389.

6. Otwinowski Z, Minor W. Processing of X-ray diffraction data in oscillation mode. Methods Enzymol 1997;276:307-326.

7. Navaza J. AMoRe: an automated package for molecular replacement. Acta Crystallogr D Biol Crystallogr 1994;50:157-163.

8. McRee DE. XtalView/Xfit—a versatile program for manipulating atomic coordinates and electron density. J Struct Biol 1999;125: 156-165.

9. Brünger AT, Adams PD, Clore GM, DeLano WL, Gros P, GrosseKunstleve RW, Jiang JS, Kuszewski J, Nilges M, Pannu NS, Read RJ, Rice LM, Simonson T, Warren GL. Crystallography and NMR system: a new software suite for macromolecular structure determination. Acta Crystallogr 1998;54:905-921. 
10. Kraulis PJ. MOLSCRIPT: a program to produce both detailed and schematic plots of protein structures. J Appl Crystallogr 1991;24:946-950.

11. Merrit EA, Bacon DJ. Raster3D: photorealistic molecular graphics. Methods Enzymol 1997;277:505-524.

12. Nicholls A, Sharp K, Honig B. Graphical representation and analysis of structural properties. Proteins 1991;11:281-296.

13. Holm L, Sander C. Touring protein fold space with Dali/FSSP. Nucleic Acids Res 1998;26:316-319.

14. Gray CH, Good VM, Tonks NK, Barford D. The structure of the cell cycle protein Cdc14 reveals a proline-directed protein phosphatase. EMBO J 2003;22:3524-3535.

15. Song H, Hanlon N, Brown NR, Noble ME, Johnson LN, Barford D. Phosphoprotein-protein interactions revealed by the crystal structure of kinase-associated phosphatase in complex with pohosphoCDK2. Mol Cell 2001;7:615-626.

16. Yuvaniyama J, Denu JM, Dixon JE, Saper MA. Crystal structure of the dual specificity protein phosphatase VHR. Science 1996:272:1328-1331.

17. Guex N, Peitsch MC. SWISS-MODEL and the Swiss-PdbViewer: an environment for comparative protein modeling. Electrophoresis $1997 ; 18: 2714-2723$

18. Jia Z, Barford D, Flint AJ, Tonks NK. Structural basis for phosphotyrosine peptide recognition by protein tyrosine phosphatase 1B. Science 1995;268:1754-1758.
19. Denu JM, Lohse DL, Vijayalakshmi J, Saper MA, Dixon JE. Visualization of intermediate and transition-state structures in protein-tyrosine phosphatase catalysis. Proc Natl Acad Sci USA 1996;93:2493-2498.

20. Denu JM, Dixon JE. Protein tyrosine phosphatases: mechanisms of catalysis and regulation. Curr Opin Chem Biol 1998;2:633-641.

21. Yoon TS, Jeong DG, Kim JH, Cho YH, Son JH, Lee JW, Ryu SE, Kim SJ. Crystal structure of the catalytic domain of human VHY, a dual-specificity protein phosphatase. Proteins 2005;61:694-697.

22. Lee JO, Yang H, Georgescu MM, Di Cristofano A, Maehama T, Shi Y, Dixon JE, Pandolfi P, Pavletich NP. Crystal structure of the PTEN tumor suppressor: implications for its phosphoinositide phosphatase activity and membrane association. Cell 1999; 99:323-334.

23. Chu HM, Guo RT, Lin TW, Chou CC, Shr HL, Lai HL, Tang TY, Cheng KJ, Selinger BL, Wang AH. Structures of Selenomonas ruminantium phytase in complex with persulfated phytate: DSP phytase fold and mechanism for sequential substrate hydrolysis. Structure 2004;12:2015-2024.

24. Sarmiento M, Puius YA, Vetter SW, Keng YF, Wu L, Zhao Y, Lawrence DS, Almo SC, Zhang ZY. Structural basis of plasticity in protein tyrosine phosphatase $1 \mathrm{~B}$ substrate recognition. Biochemistry 2000;39:8171-8179.

25. Smith SC, Kennelly PJ, Potts M. Protein-tyrosine phosphorylation in the Archaea. J Bacteriol 1997;179:2418-2420. 